\title{
Taking KLF9 to "Cort" for crimes against metabolism
}

\author{
David R. Sweet, ${ }^{1,2}$ Liyan Fan, ${ }^{1,2}$ and Mukesh K. Jain' \\ ${ }^{1}$ Case Cardiovascular Research Institute and University Hospitals Cleveland Medical Center, Cleveland, Ohio, USA. ${ }^{2}$ Department of Pathology, Case Western Reserve University, Cleveland, Ohio, USA.
}

\begin{abstract}
Glucocorticoids (GCs) are essential for proper glycemic control, but in excess, can lead to hyperglycemia and diabetes. In this issue of the JCI, Cui et al. elucidate a mechanism by which GCs regulate gluconeogenesis utilizing the transcription factor Krüppel-like factor 9 (KLF9) in physiology and disease settings. They report that KLF9 is a CC-inducible factor that ultimately increases the transcription of proliferator-activated receptor $\gamma$ coactivator 1 $\alpha$ (PCC1 $\alpha$ ), resulting in gluconeogenesis. Given the high incidence of CCinduced diabetes, identification of this signaling axis provides, not only critical scientific insight, but also a foundation for preventative therapies for patients receiving chronic GC treatment.
\end{abstract}

\section{Glucocorticoid control of glucose homeostasis: missing links for master regulators}

Glucocorticoids (GCs) are critical for proper metabolic adaptation in response to various physiologic (e.g., circadian cues) and stress stimuli. In response to fasting, the adrenal glands release GCs, which facilitate and coordinate glucose mobilization, peripheral lipolysis, and amino acid catabolism to ensure an adequate fuel supply for tissues (1). While this physiologic response is critical, excess or prolonged exposure to GCs can lead to metabolic dysfunction. For example, chronic treatment with GCs, such as dexamethasone, can lead to an iatrogenic Cushinglike syndrome, characterized by the development of pathologies such as cardiovascular disease (e.g., hypertension), osteoporosis, and metabolic disease (e.g., obesity, diabetes, and dyslipidemia). One metaanalysis reports that patients taking GCs develop hyperglycemia at a rate of $32.3 \%$ and GCinduced diabetes mellitus (GIDM) at a rate of $18.6 \%$ (2). This is likely mediated by GCs' effects on multiple levels of glucose metabolism, including impaired insulin-sensitive
GLUT4-mediated glucose uptake by skeletal muscle, enhanced protein catabolism, and fatty acid release, and induction of the hepatic gluconeogenic program (3-6).

Given the importance of hepatic gluconeogenesis on glycemic regulation, it is not surprising that both physiological and pathological GC-induced gluconeogenesis have been extensively studied. In brief, GCs augment the supply of gluconeogenic substrates by inducing protein (particularly branched-chain amino acids [BCAAs]) metabolism in muscle to liberate carbons in the form of alanine that are utilized in the liver for glucose production (3-5). In addition, GCs directly affect transcription of numerous genes involved in hepatic gluconeogenesis. GCs bind to the GC receptor (GR), which then translocates to the nucleus to bind to GC response elements on the promoters of gluconeogenic genes, including Pck1, Fbp1, and G6pc (6). GCs interact with other important transcription factors, such as forkhead box $\mathrm{O} 1$ (FoxO1) and proliferator-activated receptor $\gamma$ coactivator $1 \alpha(\mathrm{PGC} 1 \alpha)$, to modulate transcriptional activity and subsequent

Related Article: p. 2266

Conflict of interest: The authors have declared that no conflict of interest exists.

Copyright: $\odot$ 2019, American Society for Clinical Investigation.

Reference information: J Clin Invest. 2019;129(6):2178-2180.

https://doi.org/10.1172/JCI128481.

glucose handling (1). Importantly, there is a reciprocal interaction between GCs and PGC1 $\alpha$ : PGC1 $\alpha$ can coactivate the GR and other gluconeogenic transcription factors, and GC administration can increase Pgc1a transcription (7). This implicates the GC/ PGC1 $\alpha$ relationship as essential for gluconeogenesis; however, the exact mechanism of this regulation is unclear. GCinduced hyperglycemia seems to result from GR-mediated transcription of gluconeogenic genes along with the transcription of its own coactivators, such as PGC1 $\alpha$.

In this issue of the JCI, Cui and colleagues introduce a critical role of the transcription factor Krüppel-like factor 9 (KLF9) in linking GC signaling to gluconeogenesis (8). Through the use of gain- and loss-offunction experiments in hepatocytes and in multiple murine models, they establish that fasting-induced and exogenously administered GCs induce transcription of hepatic Klf9, which then augments Pgcla and, ultimately, the transcription of gluconeogenic and lipid oxidation genes. The discovery of this GR/KLF9/PGC1a axis has important implications for GIDM. Further, this work adds important information to the growing appreciation that KLFs are central mediators of metabolism.

\section{The KLFs coordinate interorgan metabolic crosstalk}

The first link between the KLF gene family and metabolism was established nearly two decades ago (9). Subsequent work has established KLFs as critical effectors of diverse metabolic processes ranging from nutrient acquisition to substrate utilization and energetics. The KLFs constitute a family of $18 \mathrm{C} 2 \mathrm{H} 2$ zinc finger transcription factors that are dynamically expressed in metabolic tissues and coordinate the handling of the nutrient class in response to various dietary and hormonal signals (reviewed in ref. 10).

In addition to regulating organ-specific utilization and storage of nutrients, KLFs affect systemic metabolic homeostasis by fine-tuning nutrient trafficking between organs in response to changes in energy status. This concept is perhaps best exemplified 


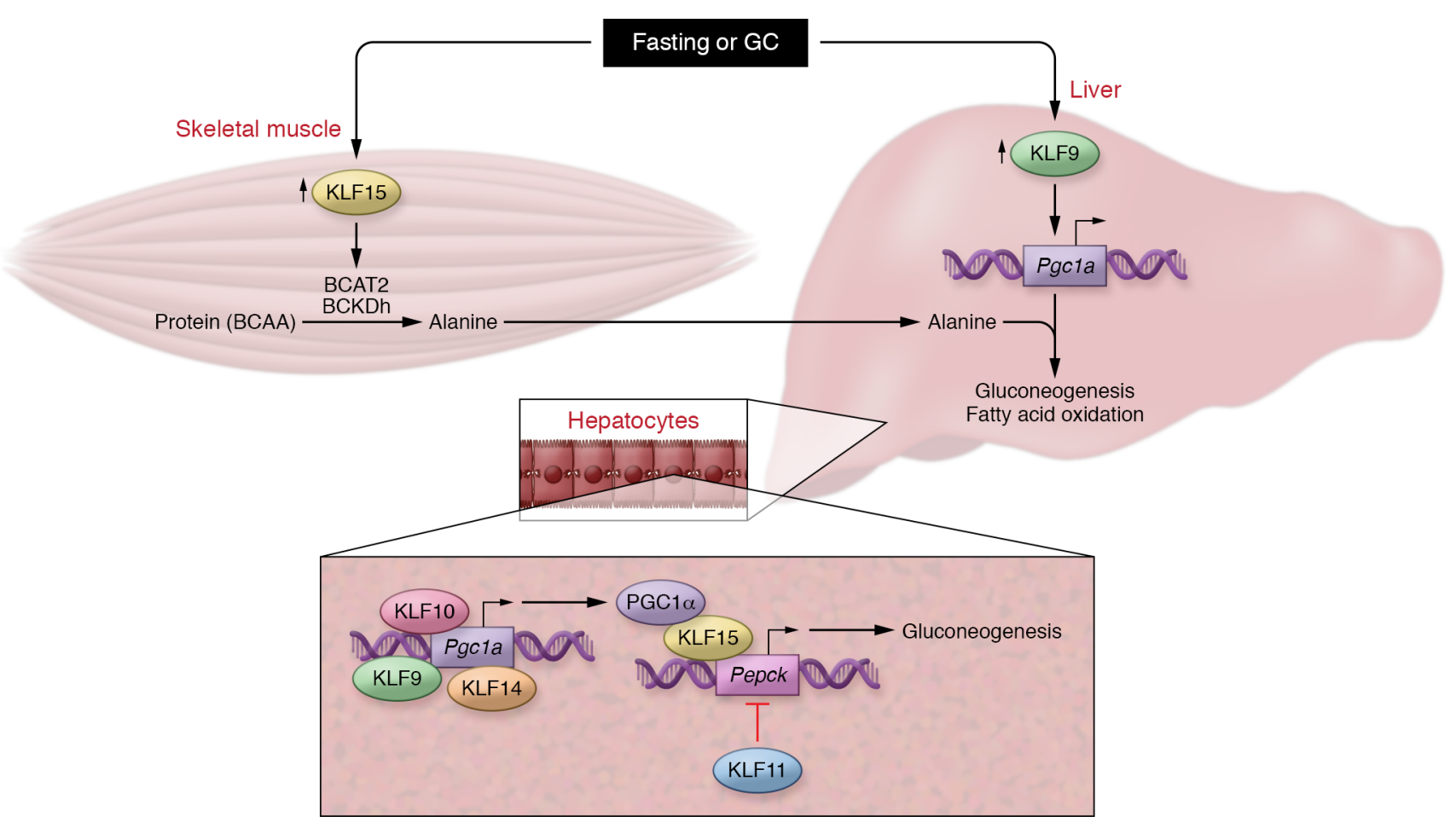

Figure 1. KLF9 participates in a KLF metabolic network. Upper panel: Proposed model by which fasting or GC release concurrently increases expression of skeletal muscle KLF15 and hepatic KLF9 to coordinate gluconeogenesis. KLF15 facilitates BCAA catabolism to provide carbon substrates (alanine) for gluconeogenesis via induction of BCAT2 and BCKDh, while KLF9 increases transcription of the gluconeogenic coactivator Pgc1a. Lower panel: Redundancy in hepatic KLF function preserves glycemic control during fasting. KLF9, KLF10, and KLF14 increase transcription of Pgc1a, while KLF15 serves as a cofactor to increase transcription of the rate-limiting enzyme Pepck. Conversely, KLF11 represses Pepck transcription, thereby inhibiting gluconeogenesis in fed states.

by KLF15 activity that regulates both lipolysis and gluconeogenesis during the fasting response. With respect to gluconeogenesis, KLF15 promotes transcription of critical enzymes in the catabolism of skeletal muscle BCAAs, including branched-chain amino acid transferase 2 (Bcat2) and the branchedchain ketoacid dehydrogenase (BCKDh) complex (11-13). Newly liberated BCAA carbon skeletons then circulate to the liver, thereby contributing to the gluconeogenic carbon substrate pool. Paired with Cui et al.'s findings on hepatic KLF9, this phenomenon illustrates a coordinated interorgan KLF network regulating a specific metabolic process (Figure 1). Cui et al. note that, upon fasting, endogenously released GCs induce hepatic KLF9, which subsequently enhances gluconeogenesis by binding to the promoter of Pgc1a. Thus, coexpression of these phylogenetically conserved factors in response to a physiologic stimulus facilitates proper systemic glycemic control. As KLF15 is also highly induced by GCs (14-16), it is likely that the induction of both KLF9 and KLF15 is critical in GIDM. However, the observation that KLF15 levels in the liver are unresponsive to GC treatment and to gain or loss of Klf9 expression suggests that regulation of KLF expression can be tissue specific, a phenomenon that could be leveraged in future studies on modulating the GC response.

\section{Hepatic KLFs provide redundancy to preserve metabolic homeostasis}

The results from Cui et al. provide exciting insights into a coordinated KLF response to fasting and GCs and also demonstrate an important facet of KLF biology: redundancy of factors to protect processes critical for organismal survival. For example, KLF2 and - 4 function in concert to preserve endothelial integrity such that one factor is able to compensate for the complete loss of the other, preventing embryonically lethal vasculature leakage (17). Given the importance of tight metabolic homeostasis, the presence of redundant control is perhaps not surprising. This is indeed the case, with the liver serving as a helpful model. In response to fasting, KLF10, KLF14, and KLF15 are all induced within the liver and promote gluconeogenesis through increasing the transcription of the rate-limiting enzyme for gluconeogenesis, PEPCK (10). Additionally, each of these factors does so via regulation of PGC1 $\alpha$ transcription or activity (Figure 1B): KLF9, KLF10, and KLF14 increase Pgc1a transcription, and KLF15 binds to and cooperates with PGC1 $\alpha$ on the Pepck promoter (18-20).

It is also noteworthy that certain KLFs have functions antagonist to their family members. For example, KLF11 serves largely to inhibit gluconeogenesis through its inhibition of Pepck transcription; thus, during states of fasting, KLF11 expression is low (21). Although Cui et al. have demonstrated that KLF9 does not affect the expression of KLF10 or KLF15, further studies exploring a potential role of KLF9 in repressing KLF11 as a means of enhancing gluconeogenesis will glean important insight into the coordination of KLF crosstalk within the liver.

\section{Perspectives and future directions}

There has been a dearth of literature regarding the metabolic functions of 
KLF9. With KLF9's newfound importance as a regulator of GC-induced hyperglycemia, exciting new avenues of research are now opened for exploration that will contribute to the rapidly growing knowledge base on KLF-centric metabolic control. For example, it will be important to establish the role circadian rhythmicity plays on hepatic KLF9 function. Circadian oscillations serve to coordinate internal molecular activity with external environmental cues (e.g., nutrient availability), and the association between disrupted circadian rhythms and metabolic disease is well established. Indeed, the gluconeogenic factors KLF10 and KLF15 exhibit circadian rhythmicity, allowing for the matching of glycemic control to feeding patterns (12, 22). A study by Spörl et al. demonstrated that epidermal KLF9 exhibits circadian oscillations in response to cortisol release; although not the focus of the work, they also demonstrated rhythmicity of hepatic KLF9 (23). Combined with the well-documented oscillatory nature of GC release, future studies investigating circadian control of KLF9's metabolic functions will advance our understanding of molecular regulators of metabolic disease.

Within this study, Cui et al. provide the first evidence of KLF9 as a prospective target for preventing GIDM. One caveat that would prevent KLF9 inhibition as a therapy for GIDM is the phenomenon that loss of hepatic KLF9 contributes to a nonalcoholic fatty liver disease (NAFLD) phenotype via the repression of fatty acid oxidation genes; however, this will require further study. Additionally, KLF9 plays a role in orchestrating GC-induced antiinflammatory transcription in macrophages (24) and serves to maintain B cell quiescence (25). Therefore, further work will need to be performed to investigate how manipulation of KLF9 will affect the well-recognized antiinflammatory effects of GCs and attendant off-target effects.

In summary, this study demonstrates that KLF9 is a key intermediate between GR and PGC1 $\alpha$ and a critical component of the signaling axis utilized by GCs to promote gluconeogenesis in both physiology (fasting) and disease (chronic GC exposure). This work contributes to growing evidence that the KLFs are central regulators of nutrient homeostasis and provides a foundation for investigations into how KLFs intersect with various metabolic signaling molecules and pathways to control whole body metabolism.

\section{Acknowledgments}

This work was funded by $\mathrm{NIH}$ grants R35HL135789/R01DK111468-01 (to MKJ), T32GM007250 (to DRS and LF), T32HL134622 (to LF), and F3OHL139014 (to DRS).

Address correspondence to: Mukesh K. Jain, Iris \& Bert L. Wolstein Research Building, 2103 Cornell Road, Cleveland, Ohio 44106, USA. Phone: 216.368.3391; Email:mxj84@case.edu.

1. Vegiopoulos A, Herzig, S. Glucocorticoids, metabolism, and metabolic diseases. Mol Cell Endocrinol. 2007;275(1-2):43-61.

2. Liu XX, et al. Hyperglycemia induced by glucocorticoids in nondiabetic patients: a meta-analysis. Ann Nutr Metab. 2014;65(4):324-332.

3. Weinstein SP, Wilson CM, Prisker A, Cushman SW. Dexamethasone inhibits insulin-stimulated recruitment of GLUT4 to the cell surface in rat skeletal muscle. Metabolism. 1998;47(1):3-6.

4. Lofberg E, et al. Effects of high doses of glucocorticoids on free amino acids, ribosomes and protein turnover in human muscle. Eur JClin Invest. 2002;32(5):345-53.

5. Felig P, Pozefsk T, Marlis E, Cahill GF. Alanine: key role in gluconeogenesis. Science. 1970;167(3920):1003-1004

6. Kuo T, McQueen A, Chen TC, Wang JC. Regulation of glucose homeostasis by glucocorticoids. In Wang JC, Harris C, eds. Glucocorticoid Signaling: Advances In Experimental Medicine And Biology. Vol 872. New York, New York, USA: Springer; 2015.

7. Yoon JC, et al. Control of hepatic gluconeogenesis through the transcriptional coactivator PGC-1. Nature. 2001;413(6852):131-138.

8. Cui A, et al. Dexamethasone-induced Krüppellike factor 9 expression promotes hepatic gluconeogenesis and hyperglycemia. J Clin Invest. 2019;129(6):2266-2278.

9. Gray S, et al. The Krüppel-like factor KLF15 regulates the insulin-sensitive glucose transporter
GLUT4. J Biol Chem. 2002;277(37):3432234328.

10. Hsieh PN, Fan L, Sweet DR, Jain MK. The Krüppellike factors and control of energy homeostasis. Endocr Rev. 2019;40(1):137-152.

11. Gray S, et al. Regulation of gluconeogenesis by Krüppel-like factor 15. Cell Matab. 2007;5(4):305-312.

12. Jeyaraj D, et al. Klf15 orchestrates circadian nitrogen homeostasis. Cell Metab. 2012;15(3):311-323.

13. Fan L, Hsieh PN, Sweet DR, Jain MK. Krüppellike factor 15: regulator of BCAA metabolism and circadian protein rhythmicity. Pharmacol Res. 2018;130:123-126.

14. Morrison-Nozik A, et al. Glucocorticoids enhance muscle endurance and ameliorate Duchenne muscular dystrophy through a defined metabolic program. Proc Natl Acad Sci U S A. 2015;112(49):E6780-E9789.

15. Asada M, et al. DNA binding-dependent glucocorticoid receptor activity promotes adipogenesis via Krüppel-like factor 15 gene expression. Lab Invest. 2011;91(2):203-215.

16. Haldar S, et al. Krüppel-like factor 15 regulates skeletal muscle lipid flux and exercise adaptation. Proc Natl Acad Sci US A. 2012;109(17):6739-6744.

17. Sangwung P, et al. KLF2 and KLF4 control endothelial identity and vascular integrity. JCI Insight. 2017;2(4):e91700.

18. Yang X, et al. KLF10 transcription factor regulates hepatic glucose metabolism in mice. Diabetologia. 2017;60(12):2443-2452.

19. Wang L, et al. The KLF14 transcription factor regulates hepatic gluconeogenesis in mice. J Biol Chem. 2017;292(52):21631-21642.

20. Takashima M, et al. Role of KLF15 in regulation of hepatic gluconeogenesis and metformin action. Diabetes. 2010;59(7):1608-1615.

21. Zhang $\mathrm{H}$, et al. Involvement of KLF11 in hepatic glucose metabolism in mice via suppressing of PEPCK-C expression. PLoS One. 2014;9(2):e89552.

22. Guillaumond F, et al. Krüppel-like factor KLF10 is a link between the circadian clock and metabolism in liver. Mol Cell Biol. 2010;30(12):3059-3070.

23. Spörl F, et al. Krüppel-like factor 9 is a circadian transcription factor in human epidermis that controls proliferation of keratinocytes. Proc Natl Acad Sci U S A. 2012;109(27):10903-10908.

24. Chinenov Y, et al. Glucocortioid receptor coordinates transcription factor-dominated regulatory network in macrophages. BMC Genomics. 2014;15:656.

25. Good KL, Tangye SG. Decreased expression of Krüppel-like factors in memory B ells induces the rapid response typical of secondary antibody responses. Proc Natl Acad Sci U S A. 2007;104(33):13420-13425. 